dium entaillé postérieurement en trapèze à còtés rectilignes, le fond de l'échancrure avec une petite coupure triangulaire médiane à peine marquée, les lobes latéraux émoussés au sommet. - Femelle inconnue.

Valachie : Comana (A.-C. Montandon). - Collections P u ton, de Vauloger, L.von Hey den et la mienne.

Cette rare espèce, la deuxième du genre, ressemble beaucoup à sa congénère portugaise, Ph. Metzneri Schauf. ( ${ }^{1}$. Elle en diffère notamment par son prothorax moins allongé, arrondi antérieurement en demi-cercle au lieu d'être atténué en ogive, par son pygidium entaillé en trapèze et non en accolade, et surtout par ses élytres granuleux, à aspect râpeux, tandis qu'ils sont simplement rugueux chez $\mathrm{Ph}$. Metzneri.

Je me fais un plaisir de la dédier à l'habile entomologiste qui l'a découverte et qui a bien voulu en enrichir ma collection par l'entremise de mon ami M, le $\mathrm{D}^{\mathrm{r}} \mathrm{Put}$.

\title{
Note sur le genre Pristaulacus Kieff. [HYMÉN.]
}

\section{Par J.-J. KiefFer.}

Dans une récente étude sur les Évaniides (Ann. Soc. ent. Fr., 1899, p. 813), j'ai séparé du genre Aulacus les espèces qui ont les crochets des tarses pectinés, et j'ai ètabli pour elles le genre Pristaulacus. J'ai rangé dans ce nouveau genre les quatre espèces suivantes : bimaculatus K i ff., Clapowskii Kieff., Morawitzi Sem. et Patrati S erv.

M. Kohl, conservateur au Musée de Vienne (Autriche), a eu l'obligeance d'examiner les Aulacus de la collection de Schletterer confiés à ses soins et de m'en communiquer le résultat. D'après ses observations, les espèces qui suivent font encore partie du genre Pristaulacus : Abbotii Westw. (Amérique du Nord), ambiguus Schlett. (Brésil), cingulatus Westw. (Australie), flavoguttatus Westw. (Australie), gloriator F ab r. (Europe), magnificus $\mathrm{S} c \mathrm{hl}$ lt. (Ceylan), melleus Cress. (Amérique du Nord), rubidus Sc hlett. (Australie) et stephanoides Westw. (Brésil). Le genre Aulacus comprend : striatus Jur. qui en est le type, fusiger Schlett., ruftarsis Cr., auxquels j'ajoute sibiricola Sem.; ces deux dernières espèces diffèrent du type par les

(1) Nunquam otiosus, I, p. 61. - Voir aussi Ern. Olivier : Essai d'une Revision des espèces européennes et circa-méditerranéennes de la famille des Lampyrides (L'Abeille, XXII, 1884, p. 37 et pl. I, fig. 8). 
crochets des tarses munis de deux petites dents et non point d'une dent unique.

Quant aux douze espèces qui suivent, et qui ne sont pas conservées au Musée de Vienne, j’ignore encore dans lequel des deux genres il faut les classer : ater Westw. (Brésil et Mexique), bicornutus Schlett. (1) (Brésil. conservé au Musée de Budapest), capitalis Schlett. (Chili, conservé au Musée de.Berlin), concolor Schlett. (Brésil), cordatus Schlett. (Australie, conservé dans la collection de M. de Saussure à Genève), Esenbecki Dahlb. (Europe), formosus Westw. (Australie), haemorrhoidellus Westw. (Brésil), Kohli Schlett. (Guyane, conservé au Musée de Genève), maculatus Schlett. (Brésil, collection de M. W üstner, à Sonderburg, île d'Alsen), pediculatus Schlett. (Brésil, conservé au Musée de Dresde) et spinifer Westw. (conservé au Musée de Stuttgart).

M. A shmead m'a écrit, que parmi les Aulacus conservésà Washington, plusieurs espèces rentrent dans le genre Pristaulacus.

\section{Description d'un Aulax nouveau [Hуме́s.]}

\section{Par J.-J. KiefFer.}

Aulax Andrei, n. sp. - Noir; abdomen d'un brun marron, plus sombre sur le dessus; funicule d'un brun rouge; pattes d'un rouge brunâtre à cuisses plus sombres; hanches noires. Antennes de 13 articles; le second article presque deux fois aussi long que gros; le $3^{\mathrm{e}}$ et le $4^{\mathrm{e}}$ égaux, trois fois aussi longs que gros et un peu plus longs que le $\breve{\partial}^{\mathrm{e}}$; le $12^{\mathrm{e}}$ de moitié plus long que gros; le $13^{\mathrm{e}}$ deux fois aussi long que l'avant-dernier. Mésonotum luisant, finement chagriné, avec deux sillons parapsidaux peu distincts et n'atteignant pas le bord antérieur. Ailes a bords ciliés; cellule radiale fermée, trois fois aussi longue que large. OEufs cylindriques, trois fois et demie aussi longs que gros et un peu plus longs que leur pédicule. Taille $\subsetneq$ : 1,50 mill. - Mâle inconnu.

Patrie. Environs de la Main-du-Prince, non loin de Bitche.

Mours et parasites. Cet Insecte, que je dédie à M. Ern. André, de Gray, produit sur la nervure médiane des feuilles d'Hypochoeris maculata L. des renflements peu apparents, ordinairement au nombre de trois à huit, et contenant chacun une loge larvaire. L'insecte en sort

(1) M. le $\mathbf{D}^{r} \mathbf{C}$. von Kertesz, conservateur au Musée de Budapest, vient de m'écrire que $A$. bicornutus $\mathrm{S}$ chlett. rentre également dans le genre Pristaulacus. 


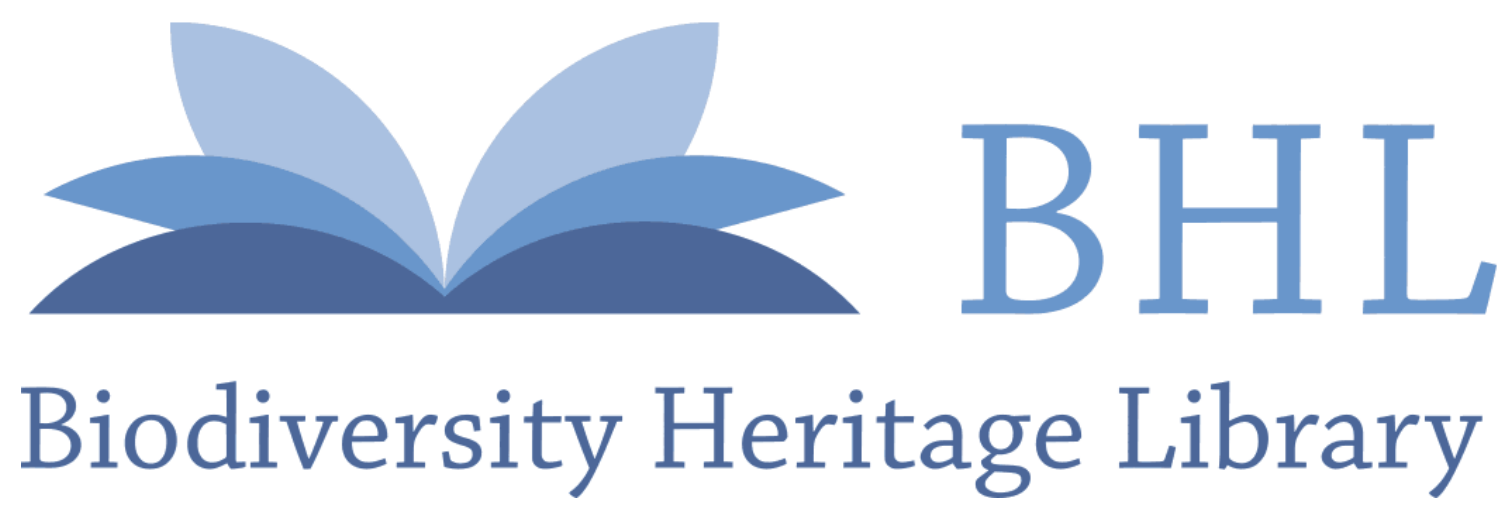

Kieffer, J.-J. 1900. "Note sur le genre Pristaulacus Kieff [Hymén]." Bulletin de la Société entomologique de France 1900, 338-339.

View This Item Online: https://www.biodiversitylibrary.org/item/36399

Permalink: https://www.biodiversitylibrary.org/partpdf/37069

\section{Holding Institution}

Smithsonian Libraries

\section{Sponsored by}

Smithsonian

\section{Copyright \& Reuse}

Copyright Status: NOT_IN_COPYRIGHT

This document was created from content at the Biodiversity Heritage Library, the world's largest open access digital library for biodiversity literature and archives. Visit BHL at https://www.biodiversitylibrary.org. 